\title{
Estudo retrospectivo de casuística das dermatofitoses em cães e gatos atendidos no Serviço de Dermatologia da Faculdade de Medicina Veterinária e Zootecnia da Universidade de São Paulo
}

Retrospective survey of dermatophytosis in dogs and cats attended at the Serviço de Dermatologia da Faculdade de Medicina Veterinária e Zootecnia da Universidade de São Paulo

\author{
Ana Claudia Balda ${ }^{1}$, Carlos Eduardo Larsson ${ }^{1}$, Mary Otsuka' ${ }^{1}$ \& Walderez Gambale ${ }^{2}$
}

\begin{abstract}
RESUMO
As dermatofitoses dos carnívoros domésticos são infecções fúngicas superficiais, causadas habitualmente por dois gêneros fúngicos: Microsporum sp. e Trichophyton sp. Trata-se de uma antropozoonose, com importância na saúde pública. O objetivo do trabalho foi caracterizar a população de cães e gatos acometidos por dermatofitose atendidos no Serviço de Dermatologia do HOVET/USP num período de 27 meses. Foram atendidos 76 animais com diagnóstico de dermatofitose (47,3\% da espécie felina e 52,7\% à canina). O agente etiológico isolado preponderantemente foi Microsporum canis. Não se observou distribuição sazonal. Os cães de raça definida foram os mais acometidos (75,0\%), mormente os Yorkshire Terrier (23,3\%). Os felinos sem e com definição racial (Persas-93,7\%) foram igualmente acometidos. Observou-se que a maioria dos animais infectados apresentava menos de um ano de idade $(65,8 \%)$. As lesões mais observadas foram: alopecia, eritema, escamas e crostas. A maioria das lesões apresentava configuração circular e estavam localizadas nas regiões cefálicas, de tronco e de membros. O prurido esteve ausente em 50,0\% dos caninos e em 88,8\% dos felinos.
\end{abstract}

Descritores: dermatofitose, Microsporum sp., Trichophyton sp. cães, gatos.

\begin{abstract}
Dermatophytosis in domestic carnivorous are superficial infections caused mainly by two genus of fungus: Microsporum $s p$ and Trichophyton sp. This disease is an anthropozoonosis important for public health. The goal of this study was characterize the population of cats and dogs with dermatophytosis treated in the Dermatology Service of HOVET - FMVZ/USP in a period of 27 months. Seventy six animals (47.3\% were felines and 52.7\% were canines) were evaluated in this study. The more frequent isolated etiological agent in canines and felines was Microsporum canis. Seasonality was not observed. The dogs with a defined breed were more predisposed (75.0\%) and the Yorkshire Terrier dogs had a higher proportion of positive cultures (23.3\%). The felines with or without breed definition got the same frequency, however, the Persians (93.7\%) were more predisposed among those of pure breed. It was noticed that the majority of infected animals were under one year of age $(65.8 \%)$. The most observed lesions were: alopecia, crusts, erythema and scales. The majority of the lesions had a circular form and were found in the cephalic, trunk and limb regions. There was no pruritus in $50.0 \%$ of the canines and $88.8 \%$ of the felines. The lesions were more inflammatory in dogs.
\end{abstract}

Key words: dermatophytosis, Microsporum sp., Trichophyton sp., dogs, cats. 


\section{INTRODUÇÃO}

O dermatófito isolado com maior freqüência em cães e gatos é o Microsporum canis, seguido pelo M. gypseum e Trichophyton mentagrophytes, embora ocorra variação de espécies em diferentes regiões do mundo [2,4,6,8-10,24,25,27,30]. A dermatofitose é caracterizada como uma antropozoonose, daí sua importância em saúde pública $[5,14,21,23,26]$.

Os animais da faixa etária de até doze meses parecem ter uma maior predisposição quanto à infecção por dermatófitos $[8,24,25]$. Alguns autores evidenciaram um maior acometimento de machos da espécie felina [13,23,27]. Já, outros referem inexistir predisposição sexual[24,14]. Com relação aos cães, há predisposição relacionada àqueles de raça definida, principalmente a Yorkshire [25]. Já, com relação aos felinos, ressalta-se a maior susceptibilidade dos animais da raça Persa $[22,23,27]$. A sazonalidade não exerce influência na incidência da infecção dermatofítica [12,14,27].

A alopecia é comumente observada nas dermatofitoses $[10,17,18,24,30]$. Uma grande variabilidade de lesões pode ocorrer nas dermatofitoses, podendo mimetizar inúmeras outras dermatoses [25]. Ocasionalmente, os gatos podem apresentar otite por Microsporum canis. Em São Paulo, estudou-se a microbiota do meato acústico externo de 50 gatos hígidos e , em $29 \%$ desses animais, foram isolados fungos do gênero Microsporum [1].

Os objetivos do trabalho foram caracterizar a população felina e canina, atendida no Hospital Veterinário (HOVET) da Faculdade de Medicina Veterinária e Zootecnia da Universidade de São Paulo (FMVZ/USP), com diagnóstico firmado de dermatofitose quanto: à idade, sexo, comprimento do pelame, definição racial e raça; aos agentes etiológicos mais comumente isolados em cultivo; à aparente transmissão interespécie; à sazonalidade; ao tipo, configuração, topografia das lesões e presença de prurido.

\section{MATERIAIS E MÉTODOS}

A amostragem foi composta a partir de casos de dermatofitose em carnívoros domésticos (caninos e felinos) atendidos, junto ao Serviço de Dermatologia do HOVET da FMVZ/USP, em um período de vinte e sete meses.
O levantamento da casuística foi executado através da análise de fichas de registro dos casos novos, atendidos no Serviço de Dermatologia da FMVZ/ USP, para obtenção do número de registro dos animais com suspeita de dermatofitose. Posteriormente, solicitou-se ao HOVET os prontuários desses animais e então, realizou-se levantamento minucioso dos dados ali dispostos para obtenção da espécie; definição racial e raça; idade; sexo; transmissão interespécie; sazonalidade; tipo; configuração, topografia lesional e eventual presença de prurido. Todas as lesões arroladas no levantamento encontravam-se demarcadas em um dermograma apenso às fichas.

Caracterizou-se o gênero e a espécie de dermatófitos através de cultivo micológico, realizados em sua totalidade no Setor de Micologia do Departamento de Microbiologia do Instituto de Ciência Biomédicas (ICB) da Universidade de São Paulo.

Utilizou-se o teste de duas proporções, com aproximação pela distribuição normal de probabilidades, a fim de verificar a existência de diferença estatisticamente significante entre os resultados obtidos. O nível de significância (valor crítico) adotado foi de $5 \%$ para as análises realizadas [3].

\section{RESULTADOS}

No período de janeiro de 1999 a março de 2001 (27 meses), o diagnóstico de dermatofitose foi estabelecido em 76 animais. Desses animais, $40(52,7 \%)$ da espécie canina e $36(47,3 \%)$ da espécie felina. Os 76 casos corresponderam a $1,7 \%$ do total de 4750 casos novos atendidos no Serviço neste período.

Dos 40 cães que apresentavam dermatofitose, $19(47,5 \%)$ eram machos e $21(52,5 \%)$ fêmeas. Já, com relação aos felinos, $22(61,1 \%)$ eram machos e $14(38,9 \%)$ fêmeas.

Quanto à definição racial e a raça, constatouse que, com relação aos animais da espécie canina, 10 $(25,0 \%)$ não apresentavam precisa definição racial e $30(75,0 \%)$ eram cães com raça definida. Dos 30 animais com definição racial, sete $(23,3 \%)$ eram da raça Yorkshire, quatro $(13,3 \%)$ da raça Pinscher, três (10\%) das raças Poodle, Boxer e Dachshund, e os demais 10 de raças distintas (Cocker Spaniel, Lhasa Apso, Weimaraner, Shar-Pei, Maltês, Bulldog e Schnauzer). Relativamente à espécie felina, $20(55,5 \%)$ dos animais não tinham definição racial e $16(44,5 \%)$ eram de raça definida, sendo 15 (93,7\%) da raça Persa e apenas um $(6,3 \%)$ da raça Siamês. 
Com relação ao comprimento do pelame, na espécie canina, 21 (52,5\%) dos animais tinham-no longo e $19(47,5 \%)$ eram animais de pelo curto. Dos felinos atendidos, $19(52,7 \%)$ eram de pelo curto e $17(47,3 \%)$ de pelame longo.

Com relação à faixa etária, nos cães, a idade variou de 1 a 120 meses, sendo que a média foi de 23,1 meses. Notou-se que $65,0 \%$ dos cães apresentavam menos de um ano de idade no momento do primo atendimento. Já, na espécie felina, a idade variou de 1 a 121 meses e a média etária foi de 20,5 meses. Dentre esses animais, $24(66,6 \%)$ tinham menos de 12 meses quando do surgimento das lesões dermatofíticas. A idade média de todos os animais, ou seja, cães e gatos somados, em meses, foi de 21,8 . Constatou-se que $50(65,8 \%)$ desses animais tinham menos de 12 meses de vida (Tabela 1).

Tabela 1. Caninos e felinos com diagnóstico de dermatofitose, segundo a idade em anos, quando do estabelecimento das lesões. Serviço de Dermatologia do HOVET - FMVZ/USP, São Paulo, janeiro de 1999 a março de 2001.

\begin{tabular}{lccccccccc}
\hline \multirow{2}{*}{ Idade } & \multicolumn{1}{c}{$<$} & \multicolumn{2}{c}{$\mathbf{1 - 3}$} & \multicolumn{2}{c}{$>$} & \multicolumn{2}{c}{ Total } \\
\cline { 2 - 9 } & & $\mathrm{N}$ & $\%$ & $\mathrm{~N}$ & $\%$ & $\mathrm{~N}$ & $\%$ & $\mathrm{~N}$ & $\%$ \\
\hline Caninos & 26 & 65,0 & 6 & 15,0 & 8 & 20,0 & 40 & 52,6 \\
Felinos & 24 & 66,6 & 7 & 19,5 & 5 & 13,9 & 36 & 47,4 \\
TOTAL & 50 & 65,8 & 13 & 17,1 & 13 & 17,1 & 76 & 100,0 \\
\hline
\end{tabular}

As lesões mais comumente observadas nos cães com dermatofitose foram: alopecia $(100,0 \%)$; crostas melicéricas ou hemorrágicas $(67,5 \%)$; eritema $(57,5 \%)$ e escamas $(45,0 \%)$. Outras lesões, também evidenciadas em diferentes percentís, foram: pápulas, hiperpigmentação, liquenificação, colarinhos epidérmicos e pústulas. As lesões assentavam-se mais freqüentemente na: região cefálica $(67,5 \%)$ e tronco (67,5\%). Já na espécie felina, observou-se mais freqüentemente: alopecia (91,6\%); crostas melicéricas e hemorrágicas $(61,1 \%)$ e escamas $(27,7 \%)$. Foram observadas, ainda, eritema, pápulas e hiperpigmentação. Essas lesões situavam-se mais freqüentemente, na região cefálica $(72,2 \%)$ e no tronco $(47,2 \%)$ dos felinos (Tabelas 2 e 3).

Quanto à conformação lesional, notou-se que, na espécie canina, em 75,0\% dos animais tais lesões eram circulares. Na espécie felina, 86,2\% apresentaram também lesões de mesma conformação. As Figuras 1,2 e 3 permitem a visualização de algumas das
Tabela 2. Caracterização lesional ( $n^{\circ}$ e $\%$ ) em caninos e felinos com dermatofitose. Serviço de Dermatologia do HOVET - FMVZ/USP, São Paulo, janeiro de 1999 a março de 2001.

\begin{tabular}{ccccccc}
\hline \multirow{2}{*}{ Lesões elementares } & \multicolumn{2}{c}{ Caninos } & \multicolumn{2}{c}{ Felinos } & \multicolumn{2}{c}{ Total } \\
\cline { 2 - 8 } & $\mathrm{N}$ & $\%$ & $\mathrm{~N}$ & $\%$ & $\mathrm{~N}$ & $\%$ \\
\hline Alopecia & 40 & 100,0 & 33 & 91,6 & 73 & 96,0 \\
Crostas* $^{*}$ & 27 & 67,5 & 22 & 61,1 & 49 & 64,5 \\
Eritema & 23 & 57,5 & 6 & 16,6 & 29 & 38,1 \\
Escamas & 18 & 45,0 & 10 & 27,7 & 38 & 36,8 \\
Pápulas & 10 & 25,0 & 2 & 5,5 & 12 & 15,8 \\
Hiperpigmentação & 5 & 12,5 & 2 & 5,5 & 7 & 9,2 \\
Liquenificação & 2 & 5,0 & $\tilde{\mathrm{N}}-$ & $\tilde{\mathrm{N}}-$ & 2 & 2,6 \\
Colarinho epidérmico & 1 & 2,5 & $\tilde{\mathrm{N}}-$ & $\tilde{\mathrm{N}}-$ & 1 & 1,3 \\
Pústulas & 1 & 2,5 & $\tilde{\mathrm{N}}-$ & $\tilde{\mathrm{N}}-$ & 1 & 1,3 \\
\hline *Melicéricas/hemorrágicas & & & & &
\end{tabular}

Tabela 3. Topografia das lesões cutâneas iniciais (nº e \%) dos caninos e felinos com dermatofitose. Serviço de Dermatologia do HOVET - FMVZ/USP, São Paulo, janeiro de 1999 a março de 2001.

\begin{tabular}{ccccccc}
\hline \multirow{2}{*}{ Localização } & \multicolumn{2}{c}{ Caninos } & \multicolumn{2}{c}{ Felinos } & \multicolumn{2}{c}{ Total } \\
\cline { 2 - 7 } & N & $\%$ & N & $\%$ & N & $\%$ \\
\hline Cefálica & 27 & 67,5 & 26 & 72,2 & 53 & 69,7 \\
Tronco & 27 & 67,5 & 17 & 47,2 & 44 & 57,9 \\
Membros & 17 & 42,5 & 12 & 33,3 & 29 & 38,1 \\
Cervical & 13 & 32,5 & 8 & 22,2 & 21 & 27,6 \\
Abdômen & 9 & 22,5 & 7 & 19,4 & 16 & 21,0 \\
Lombo-sacra & 6 & 15,0 & --- & --- & 6 & 7,9 \\
Coggínea & --- & --- & 7 & 19,4 & 7 & 9,2 \\
\hline
\end{tabular}

lesões, bem como de sua topografia e conformação, em três dos animais que apresentaram dermatofitose.

No que se refere à espécie de dermatófito isolada nestes 76 animais, $M$. canis foi identificado em $66(86,8 \%)$ dos animais, M. gypseum em nove (11,9\%) e T. rubrum em um (1,3\%) destes carnívoros. A distribuição dos gêneros e espécies fúngicas, de acordo com o acometimento segundo a espécie, está disposta no Gráfico 1.

Quanto a sazonalidade, observou-se que 21 animais $(27,6 \%)$ se infectaram na primavera, 20 $(26,3 \%)$ no verão, $17(22,4 \%)$ no outono e $18(23,7 \%)$ no inverno. A sazonalidade de ocorrência de acordo com a espécie infectada está disposta no Gráfico 2. 


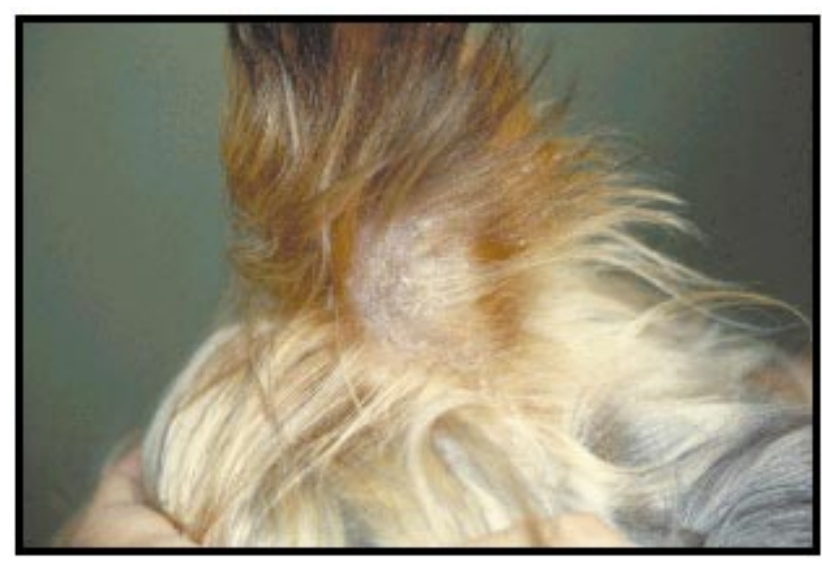

Figura 1. Dermatofitose - lesão alopécica de conformação circular, com presença de escamas e crostas melicéricas, em face externa de pavilhão auricular (canina, Yorkshire, fêmea, 8 meses, pront. 112501) HOVET/FMVZ-USP. São Paulo, 2001.

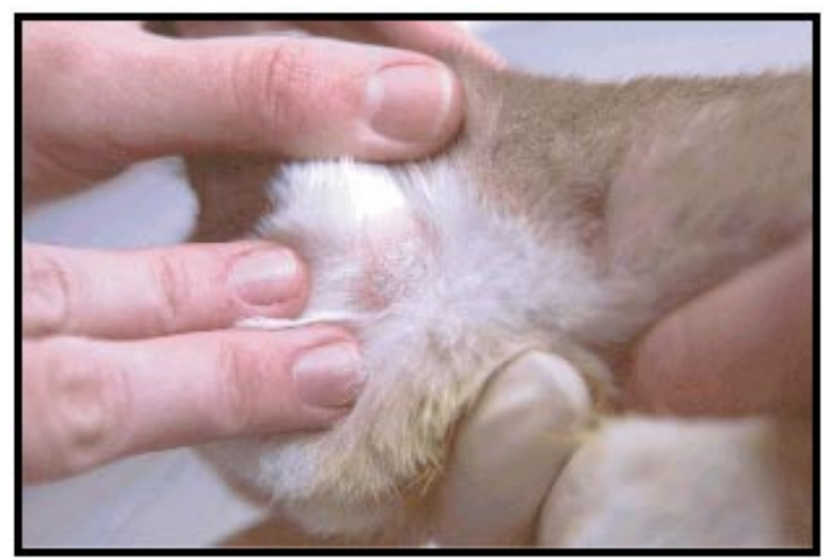

Figura 2. Dermatofitose - lesão alopécica de conformação circular, com presença de eritema encimado por crostas melicéricas, em região pré-auricular (felino, sem raça definida (SRD), macho, 11meses, pront. 108493). HOVET/FMVZUSP. São Paulo, 2001.

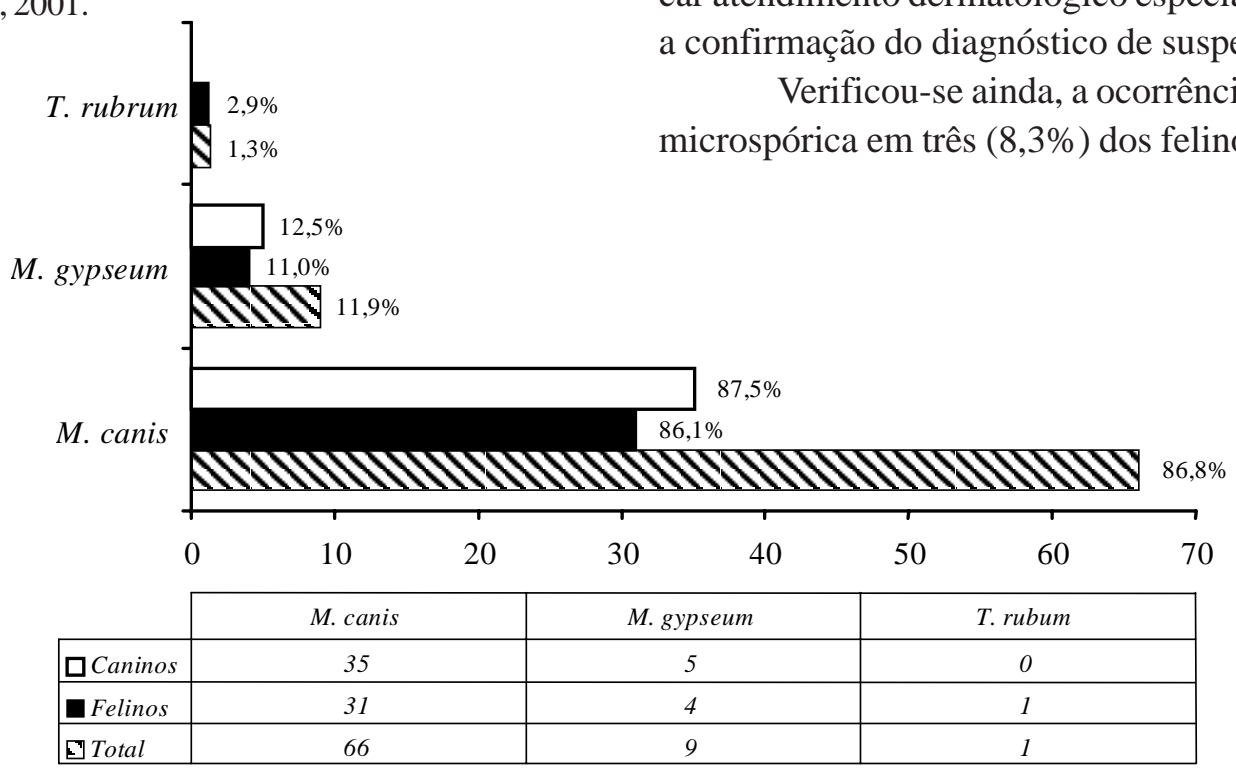

Gráfico 1. Ocorrência (№ e \%) de dermatofitose canina e felina segundo a espécie de dermatófito infectante. Serviço de Dermatologia do HOVET - FMVZ/USP (janeiro de 1999 a março de 2001).
No que concerne à manifestação sintomática representada pelo prurido, relativamente aos cães, pôde-se notar que $20(50,0 \%)$ não apresentavam prurido, dois $(5,0 \%)$ apresentavam prurido discreto e 18 (45,0\%) apresentavam-no com intensidade moderada. Já, com relação à espécie felina pôde-se observar que a maioria dos animais $(88,8 \%)$ não apresentava prurido, e que apenas quatro $(11,2 \%)$ dos animais apresentavam prurido de intensidade moderada (Tabela 4).

Relativamente à eventual transmissão interespécie, com relação aos 107 humanos que mantinham contato com os animais examinados, observouse em $23(21,5 \%)$ dos contactantes, lesões sugestivas de dermatofitose, tendo sido a eles recomendado buscar atendimento dermatológico especializado visando a confirmação do diagnóstico de suspeição.

Verificou-se ainda, a ocorrência de otomicose microspórica em três $(8,3 \%)$ dos felinos atendidos. 


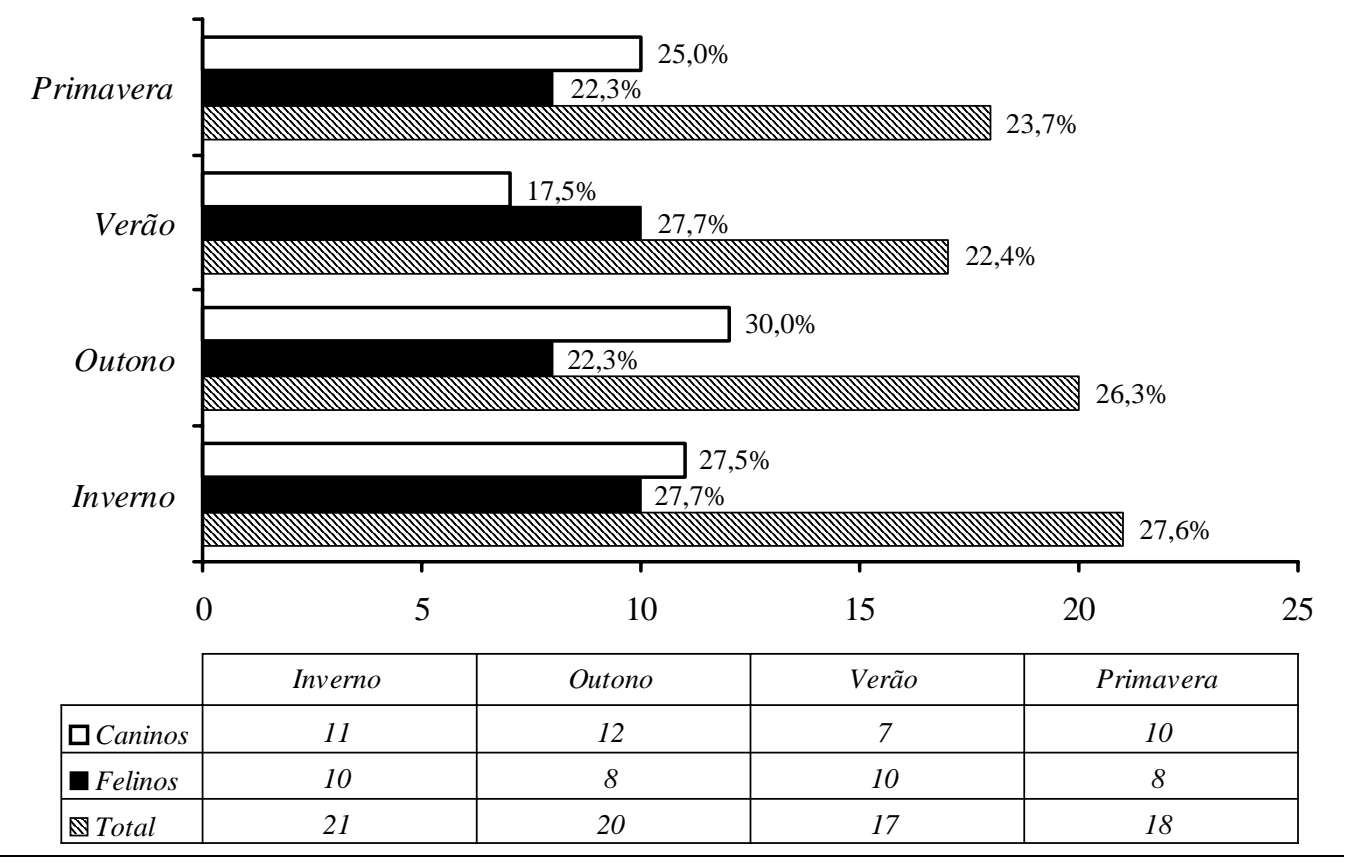

Gráfico 2. Ocorrência (№ e \%) de dermatofitose canina e felina segundo a sazonalidade. Serviço de Dermatologia do HOVET - FMVZ/USP (janeiro de 1999 a março de 2001).

\section{DISCUSSÃO}

A dermatofitose nos animais de guarda e companhia, que mantêm estreito contato com seus proprietários, se constitui em dermatopatia de importância não só pela freqüência com que é diagnosticada em todo o mundo mas, principalmente, pelo seu potencial zoonótico.

Neste trabalho, a magnitude porcentual de ocorrência $(1,7 \%)$ relativamente ao total de casos novos atendidos, em período de 27 meses de atendimento dermatológico, com relação às espécies canina e felina, coaduna com aquelas de autores estrangeiros a exemplo do evidenciado por outros autores[23], da ordem, respectivamente, de 1,2 e 3,6\% dos casos em caninos e felinos. A transmissão interespécie, ora verificada, em $21,2 \%$ dos casos de dermatofitose de cães e gatos, já vem sendo relatada ao longo dos anos por inúmeros outros pesquisadores, em todas as latitudes e com percentís de ocorrência situados entre 18,7 e $87,0 \%[5,15,19,21,29]$.

Microsporum canis, tal como o referido em literatura $[2,4,6,8-10,24,25,27,30]$ foi a espécie fúngica mais comumente encontrada nos cultivos micológicos, tanto nos animais da espécie canina como felina. Os casos de microsporose pelo M. gypseum, da ordem de $11,8 \%$, estão bem próximos em termos numéricos daqueles achados por pesquisadores brasileiros[8]. Já o Trichophyton mentagrophytes, cuja ocorrência foi relatada por autores estrangeiros $[25,27,10]$, encontrado muitas vezes com frequiência superior quando comparado ao M. gypseum [4] não foi isolado em nenhum dos 76 animais ora acompanhados. Porém, dentre os trabalhos brasileiros compulsados da bibliografia, apenas os autores mineiros[26] encontraram o $T$. mentagrophytes em freqüência equivalente àquela do M. gypseum. Os demais pesquisadores do mesmo país $[2,8,13,30]$ referem-se ao $M$. gypseum como o segundo dermatófito mais freqüente na etiologia das dermatofitoses. Merece destaque o fato de se ter isolado a partir de material provindo de um felino, cepa de Trichophyton rubrum, fungo antropofílico, cujo isolamento em animais não é corriqueiro. É bem provável que esse fungo tenha sido adquirido a partir de um contactante humano, modalidade de transmissão essa, já relatada, no Japão, há anos atrás[11]. Em cães, também já foram relatados no Brasil dois casos de tricofitose canina por este mesmo agente e com os autores concluindo que as fontes de contaminação tenham sido os proprietários [7].

No que tange à predisposição etária quanto à instalação das dermatofitoses, os percentís ora obtidos de cerca de $65,0 \%$ de acometimento de cães e gatos, da faixa etária de até doze meses de idade, coadunam com a grande maioria dos relatos dispostos 
em bibliografia $[8,23,25,26]$. Poucos autores $[29,30]$ enfocam diferenças nas faixas etárias de ocorrência de dermatofitose. É possível que esta maior susceptibilidade dos animais jovens em adquirir a infecção esteja vinculada à imaturidade do sistema imunológico.

Não se verificou predisposição sexual relacionada às infecções fúngicas superficiais, fato esse, também relatado por outros autores [12,26]. Contrapondo-se ao evidenciado no presente estudo, alguns autores observaram um maior acometimento dos machos da espécie felina $[11,26]$.

Com relação à sazonalidade, como já constatado pela maioria dos autores [12,14,27], também, neste levantamento, não se demonstrou vinculação das infecções dermatofíticas às estações do ano.

Neste estudo retrospectivo, não observou-se diferença na ocorrência da dermatofitose com relação aos felinos com e sem definição racial, entretanto, dentre os gatos que apresentavam definição racial, notouse que 15 deles $(93,7 \%)$ pertenciam à raça Persa. Tal predisposição, também foi observada por diversos outros autores [23-25]. Já com relação aos cães, nesse estudo, $75,0 \%$ dos animais possuíam raça definida, sendo que desses, praticamente um quarto $(23,3 \%)$ pertencia à raça Yorkshire. Esses dados corroboram outros estudos [25] que observaram uma maior proporção de cães com raça definida, principalmente, Yorkshire Terriers $(86 \%)$ acometidos por dermatofitose causada pelo M. canis.

É provável que, no caso dos animais com definição racial, principalmente aqueles provenientes de canís ou gatís, a aglomeração seja um fator predisponente para o contágio. A mesma suposição pode ser conjecturada para os animais da espécie felina, sem definição racial, que freqüentemente são manejados da mesma forma. Outra hipótese formulável, é a de que fatores genéticos que selecionem algum tipo de disfunção relacionado às células do sistema imune possam influenciar na susceptibilidade de determinadas raças em contraírem infecções dermatofíticas.

Com relação à topografia das lesões, notou-se que em $65,2 \%$ dos cães apresentavam lesões em região cefálica; $56,5 \%$ em região dorsal; 47,8\%, respectivamente em membros e região cervical; $34,8 \%$ em região ventral e $21,7 \%$ na região coccígea, corroborando os dados dispostos em literatura, que referem como áreas de maior acometimento, as regiões cefálica, cervical e dos membros $[21,25,30]$.
Com exceção da alopecia, lesão cutânea presente, praticamente na totalidade de casos de dermatofitose ora avaliados, e que se constitui na clássica e decantada lesão padrão das tinhas, em todos os continentes e de há muito tempo[10,16-18,24,30], não se pode cotejar os percentís de ocorrência das demais lesões com dados quantitativos de outros autores pela simples inexistência destes.

Embora em baixa magnitude de ocorrência $(8,3 \%)$ merecem ser destacados, os casos de otomicose microspórica evidenciados em felinos. Tal achado, já relatado por outros autores [1,28], não é usual. No Brasil, seguramente poucos são os clínicos veterinários informados sobre essa possibilidade etiológica de desencadeamento de otopatias, levando conseqüentemente à falhas de diagnóstico e, principalmente, ao insucesso de protocolos de terapia.

\section{CONCLUSÕES}

Os casos de dermatofitose, diagnosticados em período de 27 meses, no Serviço de Dermatologia do HOVET da FMVZ/USP:

- acometeram preponderantemente caninos e felinos com até 12 meses de idade, independentemente do sexo; foram mais freqüentes em cães de raça definida, mormente em Yorkshires, e em gatos sem raça definida;

- não eram pruriginosas na grande maioria dos casos nos felinos e quando o prurido esteve presente era de intensidade moderada;

- manifestaram-se em ambas as espécies, por lesões alopécicas, crostosas, discrômicas, descamativas, papulares e nos cães adicionalmente, pela presença de liquenificação, pústulas e de lesão em colarinho epidérmico;

- topograficamente, observaram-se lesões assestadas em ambas as espécies, em ordem decrescente de frequiência, nas regiões cefálica, torácica, de membros, cervical e abdominal;

- tiveram como agentes etiológicos mais comuns, em ambas as espécies, Microsporum canis e Microsporum gypseum, sendo que em um felino isolou-se cepa de dermatófito antropofílico (Trichophyton rubrum);

- evidenciou-se a presença de Microsporum canis nos meatos acústicos de gatos;

- inexistiu predisposição sazonal quanto à ocorrência da dermatofitose canina e felina. 


\section{REFERÊNCIAS}

1 Amaral R.C., Ibañez J.F., Mamizuka E.M., Gambale W., Paula C.R. \& Larsson C.E. 1998. Microbiota indígena do meato acústico externo de gatos hígidos. Ciência Rural. 28: 441-445.

2 Batista Júnior J.A. \& Nico F. 1967. Incidência do Microsporum canis em cães de Belo Horizonte. Arquivos da Escola de Veterinária. 19: 89-93.

3 Berquó E.S., Souza S.M.P. \& Gotlieb S.L.D. 1981. Bioestatística. São Paulo: Editora Pedagógica e Universitária, 98p.

4 Cabañes F.J., Abarca M.L. \& Bragulat M.R. 1997. Dermatophytosis isolated from domestic animals in Barcelona, Spain. Mycopathologia. 137: 107-113.

5 Cuadros J.A., Garcia, J., Alos, J.I. \& Gonzales-Placio R. 1990. Dermatophytosis in a urban setting: prospective study of 135 cases. Enfermedades Infecciosas y Microbiologia Clinica. 8: 429-433.

6 Ferreiro C.L.R., Berg V., Quadrado S.S. \& Ferreiro L. 1997. Comparação da incidência dos agentes etiológicos das dermatomicoses dos carnívoros domésticos na região da grande Porto Alegre, RS (Brasil) entre os períodos 1979-82 e 1996-97. In: Anais do XXV Congresso Brasileiro de Medicina Veterinária (Gramado, Brasil). p.15.

7 Ferreiro L., Appelt C.E., Berg V., Quadrado S.S. \& Ferreiro C.L.R. 2001. Dermatofitose canina por Trichophyton rubrum na cidade de Porto Alegre - RS, Brasil. In: III Congresso Brasileiro de Micologia (Águas de Lindóia, Brasil). p.129.

8 Gambale W., Correa B., Paul, C.R., Purchio A. \& Larsson C.E. 1987. Ocorrência de fungos em lesões superficiais de cães na cidade de São Paulo, Brasil. Revista da Faculdade de Medicina Veterinária e Zootecnia da Universidade de São Paulo. 24: 187-192.

9 Gambale W., Paula C.R., Corrêa B. \& Purchio A. 1987. Incidência de micoses superficiais em São Paulo, Capital. Anais Brasileiros de Dermatologia. 62: 193-194.

10 Jaham C. \& Paradis M. 1997. La dermatophytie féline I: étiopathogénie, aspects clinique et principes diagnostiques. Le Médecin Vétérinaire du Québec. 27: 141-155.

11 Kushida T. \& Watanabe S. 1975. Canine ringworm caused by Trichophyton rubrum; probable transmission from man to animal. Sabouraudia. 13: 30-32.

12 Larsson C.E., Lucas R. \& Germano P.M.L. 1997. Dermatofitoses de cães e gatos em São Paulo: estudo de possível influência sazonal. Anais Brasileiros de Dermatologia. 72: 139-142.

13 Larsson C.E., Nahas C.R., Ledon A.L.B.P., Gambale W., Paula C.R. \& Correa B. 1994. Ringworm in domestic cats in São Paulo, Brazil, between 1981-1990. Feline Practice. 22: 219-222.

14 Lewis D.T., Foil C.S. \& Hosgood G. 1991. Epidemiology and clinical features of dermatophytosis in dogs and cats at Louisiana State University. Veterinary Dermatology. 2: 53-58.

15 Londero A.T., Fischman O. \& Ramos C.D. 1961. Importância do gato na transmissão do Microsporum canis no Rio Grande do Sul (Brasil). Revista do Instituto de Medicina Tropical de São Paulo. 3: 81-84.

16 Moriello K.A. \& DeBoer D.J. 1994. Efficacy of systemic antifungal agents in the treatment of experimental feline dermatophytosis. In: Proceedings of the 10th Annual Meeting of the American Academy of Veterinary Dermatology and American College of Veterinary Dermatology (Charleston, U.S.A.). p.19.

17 Nesbitt G.H. \& Ackerman L. 1998. Dermotherapeutics. In: Canine and feline dermatology - diagnosis and treatment. New Jersey: ULS, pp. 71-108.

18 Pepin G.A. \& Oxenham M. 1986. Zoonotic dermatophytosis (ringworm). Veterinary Record. 118: 110-111.

19 Pinheiro A.Q., Moreira J.L. \& Sidrim J.J. 1997. Dermatophytoses in the urban environment and the coexistance of man with dogs and cats. Revista da Sociedade Brasileira de Medicina Tropical. 30: 287-294.

20 Pinter L., Jurak Z., Ukalovic M. \& Susic V. 1999. Epidemiological and clinical features of dermatophytosis in dogs and cats in Croatia between 1990 and 1998. Veterinarski Archiv. 69: 261-270.

21 Scott D.W. \& Miller Jr. W.H. 1999. Idiosyncratic cutaneous adverse drug reactions in the dog: literature review and report of 101 cases (1990-1996). Canine Practice. 24: 16-22.

22 Scott D.W., Miller W.H. \& Griffin C.E. 2001.Fungal Skin Diseases. In: Small Animal Dermatology. 6th edn. Philadelphia: Saunders, pp. 336-422. 
Balda A.C., Larsson C.E., Otsuka M.\& Gambale W.2004. Estudo retrospectivo de casuística das dermatofitoses em cães e gatos atendidos no serviço de dermatologia da Faculdade de Medicina Veterinária... $\quad$ Acta Scientiae Veterinariae. 32:133-140.

23 Scott D.W. \& Paradis M. 1990. A survey of canine and feline skin disorders seen in a university practice: Small Animal Clinic, University of Montréal, Saint-Hyancinthe, Québec (1987-1988). Canadian Veterinary Journal. 31: 830-835.

24 Smith E.K. 1988. Dermatophytosis in pets: avoiding misdiagnosis. Veterinary Medicine. 83: 554-565.

25 Sparkes A.H., Gruffydd-Jones T.J., Shaw S.E., Wright A.I. \& Stokes C.R. 1993. Epidemiological and diagnostic features of canine and feline dermatophytosis in the United Kingdom from 1956 to 1991. Veterinary Record. 133: 57-61.

26 Torres S.M.F. \& Moreira Y.K. 1994. Dermatomicoses em cães em Belo Horizonte, Minas Gerais, Brasil. Arquivos Brasileiros de Medicina Veterinária e Zootecnia. 36: 695-701.

27 Warner R.D. 1984. Ocurrence and impact of zoonoses in pet dogs and cats at USAAir Force bases. American Journal of Public Health. 74: 1239-1242.

28 Wawrzkiewicz K., Ziólkowska A.C. \& Wawrzkiewicz J. 1984. Microsporum canis: the major etiological agent of ringworm in cats and dogs. Medycyna Weterynarya. 50: 319-322.

29 Willense T. 1998. Dermatofitose. In: Dermatologia clínica de cães e gatos. 2.ed. Holanda: Elservier, pp. 22-25.

30 Yamamura A.A.M., Pereira E.P., Shimada M.K., Fugiwara C.Y., Danhone A.S. \& Chami D. 1997. Ocorrência de dermatofitose em cães e gatos atendidos pelo Hospital Veterinário da Universidade Estadual de Londrina, Paraná. Semina Ciências Agrárias. 18: 41-44. 